\title{
Sustained coherent spin wave emission using frequency combs
}

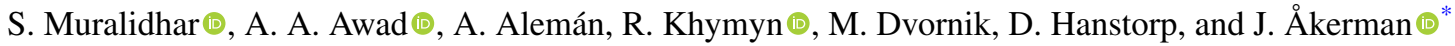 \\ Physics Department, University of Gothenburg, 41296 Gothenburg, Sweden
}

(Received 2 October 2019; revised manuscript received 16 May 2020; accepted 22 May 2020; published 15 June 2020)

\begin{abstract}
We demonstrate sustained coherent emission of spin waves in NiFe films using rapid demagnetization from high repetition rate femtosecond laser pulse trains. As the pulse separation is shorter than the magnon decay time, magnons having a frequency equal to a multiple of the $1 \mathrm{GHz}$ repetition rate are coherently amplified. Using scanning Brillouin light scattering (BLS) microscopy, we observe this coherent amplification as strong peaks spaced $1 \mathrm{GHz}$ apart. The BLS counts vs laser power exhibit a stronger than parabolic dependence consistent with counts being proportional to the square of the magnetodynamic amplitude, and the demagnetization pulse strength being described by a Bloch law. Spatial spin wave mapping demonstrates how both localized and propagating spin waves can be excited, and how the propagation direction can be directly controlled. Our results demonstrate the versatility of frequency combs and BLS spectroscopy for rapid demagnetization studies, and enable a platform for photomagnonics where sustained coherent spin waves can be utilized.
\end{abstract}

DOI: 10.1103/PhysRevB.101.224423

\section{INTRODUCTION}

Magnonics has emerged as a central research topic in nanomagnetism, with rich physics and an increasing number of novel phenomena thanks to the unique field-tunable properties of spin waves (SWs) and a wide range of metallic and insulating magnetic materials [1-4]. As the wavelength of SWs can be several orders of magnitude smaller than its electromagnetic radiation counterparts at the same frequency, the possibility of scaling down high-frequency devices using magnonics offers excellent prospects for miniaturization $[3,5]$.

SWs can be excited using a wide range of mechanisms and techniques. While the most straightforward and conventional SW generation mechanism is that of an externally applied microwave field using RF antennas [6-8], the more recent spin transfer torque and spin Hall effects generated by direct currents through nanodevices have made it possible to generate truly short-wavelength, highly nonlinear, and very high intensity SWs on the nanoscale [9-17]. SWs can also be generated optically using focused femtosecond laser (fs laser) pulses inducing rapid demagnetization [18-23] of the local magnetization, modulation of the magnetic anisotropy, thermally [24] and nonthermally [25,26], and through nonthermal optical effects such as the inverse Faraday effect [27].

Single-pulse excitation schemes, where the system relaxes back into equilibrium before the arrival of the subsequent

\footnotetext{
*johan.akerman@physics.gu.se
}

Published by the American Physical Society under the terms of the Creative Commons Attribution 4.0 International license. Further distribution of this work must maintain attribution to the author(s) and the published article's title, journal citation, and DOI. Funded by Bibsam. pulse, have been studied extensively in metals [22,28-31] and dielectrics [27,32,33]. SWs excited from an individual pulse will have damped out well before the arrival of the next pulse since the pump pulses are usually separated by about $12 \mathrm{~ns}$ or more, corresponding to a repetition rate of $80 \mathrm{MHz}$ or lower. As the typical SW decay time in ferromagnetic metals is a few nanoseconds, any attempt at generating continuous spin waves using optical means hence requires much shorter pulse separation in order to overcome the damping. The first steps toward reducing the time between consecutive pulses were taken by employing pump-probe techniques with dual pump pulses. By tailoring the time delay between two such pump pulses, the precession in both single- [26,34] and multiple-layer [35] systems could either be quenched or amplified. While carefully controlling the phase relation allowed for detailed selectivity of which SWs to excite and further amplify, the overall duty cycle was however not improved. A more accessible approach is instead to increase the fs-laser repetition rate to approach the timescale of the SW decay. As frequency comb based fs lasers with $\mathrm{GHz}$ repetition rates have recently become commercially available, the very first studies of high repetition rate SW excitations in thick extended yttrium iron garnet (YIG) films with very low damping have been reported [36,37]. The inverse Faraday effect was used for excitation with a $10 \mu \mathrm{m}$ laser spot size, and a conventional time-resolved magneto-optical Kerr effect (TR-MOKE) pump-probe technique was used for detection.

Here we demonstrate how frequency combs driving thermal rapid demagnetization can be used to excite sustained SWs in ferromagnetic metal thin films at length scales down to the laser diffraction limit. Using a unique Brillouin light scattering (BLS) microscope, where we combine a diffractionlimited BLS SW detection scheme with a diffraction-limited high repetition rate $(1 \mathrm{GHz})$ fs laser, we demonstrate continuous and directional coherent SW emission over a wide range of magnetic fields and frequencies. The high sensitivity of our 


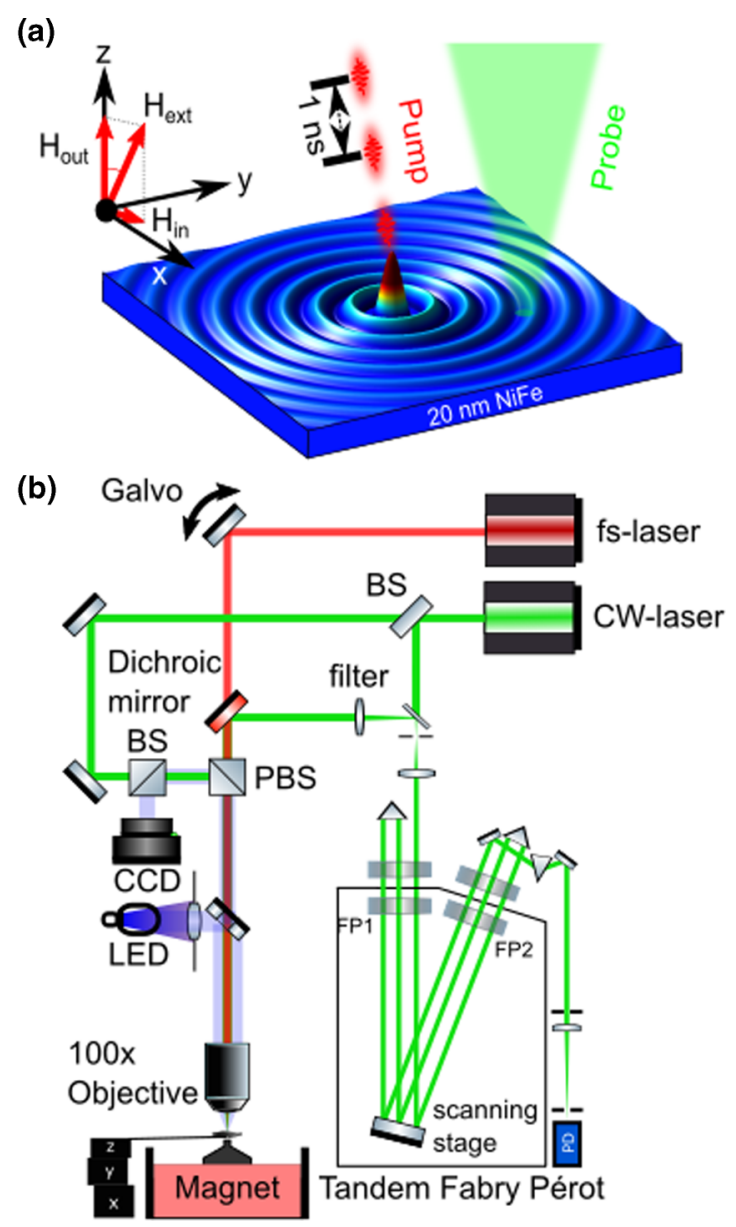

FIG. 1. Schematic of the experiment and the experimental setup. (a) The sample is pumped with a red $(816 \mathrm{~nm}) 120 \mathrm{fs}, 1 \mathrm{GHz}$ pulsed laser and probed by a continuous green laser $(532 \mathrm{~nm})$; the relative distance between the pump and the probe can be scanned over $\pm 40 \mu \mathrm{m}$. The magnetic field $H_{\mathrm{ext}}$ is applied at an oblique out-of-plane angle of $82^{\circ}$. (b) The optical setup of the pump-probe experiment: BS, 50:50 beam splitter; PBS, polarizing beam splitter. The sample is placed right below a $100 \times$ objective with NA $=0.75$ to achieve diffraction-limited focusing. The backscattered beam is analyzed using a 6-pass tandem Fabry-Pérot interferometer (TFPI) and detected with a single-channel avalanche photodiode (APD).

BLS microscope resolves both localized and propagating SWs and by varying the separation between the pump and probe laser spots, the spatial profile and direction of the SWs can be elucidated in detail. The coherent excitation of magnons is corroborated by the observation that this highly efficient SW generation only occurs at higher harmonics of the $1 \mathrm{GHz}$ laser repetition rate. In other words, only SWs that are in phase with the incoming laser pulses are coherently amplified, while all other SWs are left unaffected. When the pump laser power is varied, we observe a nonlinear, stronger than square dependence of the BLS counts, where the deviations from a square dependence can be fully accounted for by incorporating a Bloch law dependence of how the step in demagnetization field relates to the instantaneous magnon temperature [38,39]. By tuning the SW dispersion, through the magnitude and direction of the applied magnetic field, we can furthermore choose a specific wave vector to be coherently amplified, and also steer the direction of the amplified SW propagation. Our results clearly demonstrate the versatility and benefits of using frequency combs and spatially resolved BLS microscopy for the study of rapid demagnetization in ferromagnetic metals.

\section{EXPERIMENTAL DETAILS}

\section{A. Sample fabrication}

Permalloy $\left(\mathrm{Ni}_{80} \mathrm{Fe}_{20}\right)$ thin films were sputtered onto clean sapphire substrates ( $c$ plane) by dc magnetron sputtering under a 3 mTorr Ar atmosphere at ultrahigh vacuum with a $1.5 \times 10^{-8}$ mTorr base pressure. Sapphire was chosen for its negligible absorbance at the excitation laser wavelength and high thermal conductivity. The films were capped in situ with $2 \mathrm{~nm} \mathrm{SiO} 2$ to prevent oxidation of the magnetic layer.

\section{B. Frequency comb excitation and BLS microscope}

Our pump-probe experiment is shown schematically in Fig. 1(a): The pump beam was produced by a commercial Ti:Sa mode-locked laser with a $1 \mathrm{GHz}$ repetition rate at a wavelength of $816 \mathrm{~nm}$, a $30 \mathrm{fs}$ Fourier-limited pulse duration, and pulse energies up to $1 \mathrm{~nJ}$. The laser pulse stretches during propagation in the optical system [Fig. 1(b)] and reaches the sample with a duration of $120 \mathrm{fs}$. The laser was focused close to the diffraction limit using a $100 \times$ microscope objective with a NA of 0.75 . The magnetodynamics was probed using BLS microscopy [40]. A single-frequency $532 \mathrm{~nm} \mathrm{CW}$ laser was focused to the diffraction limit through the same objective as used for the pump beam. Backscattered light was collected and filtered using a polarizer and analyzed in a 6-pass tandem Fabry-Pérot interferometer (TFPI) and detected using a single channel avalanche photodiode. The optical system is equipped with a pair of galvanometer mirrors and lenses that allow the pump beam to be scanned over the sample to change the lateral distance between the pump and the probe beams.

\section{RESULTS AND DISCUSSION}

\section{A. Field and power dependent spectrum}

Figure 2(a) shows the typical field dependence of the thermal SW spectrum for the permalloy thin film sample when the pump laser is off. The sharp lower cutoff of the SW band can be clearly seen as it follows a Kittel-like frequency-vsfield dependence. A more gradual decay of the BLS counts is observed at higher SW frequencies as the SW wave vector approaches the resolution limit of the BLS. Figure 2(b) shows the corresponding spectrum at the same location when the frequency comb is turned on with a pump fluence of $1.8 \mathrm{~mJ} / \mathrm{cm}^{2}$. The spectrum changes character entirely and a number of distinct peaks appear at the harmonics of the $1 \mathrm{GHz}$ repetition rate. The strongest peaks have about an order of magnitude higher intensity than the thermal background. While the peaks fall close to the bottom of the original SW band, there are now also sizable BLS counts well below the gap at these harmonics.

To better discern all features of the laser-induced SW intensity, we show in Fig. 3 a detailed plot of the BLS counts vs frequency at a field magnitude of $600 \mathrm{mT}$ for four different 


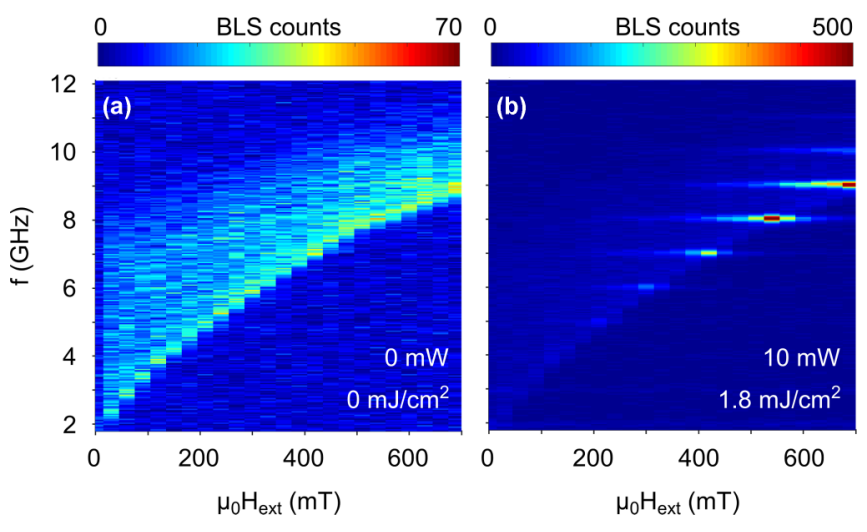

FIG. 2. Thermal and laser driven spin wave excitation in a $20 \mathrm{~nm}$ $\mathrm{NiFe}$ thin film. (a) Thermal SW spectrum vs magnetic field applied at a $82^{\circ}$ out-of-plane angle. (b) SW spectrum when the same film is irradiated with the fs-laser pulse train at a $1.8 \mathrm{~mJ} / \mathrm{cm}^{2}$ fluence.

laser powers together with the thermal SW background. We first note that $5 \mathrm{~mW}$ seems to be the approximate threshold for any noticeable additional SW intensity compared to the thermal SWs. At this lowest power level, we can observe additional BLS counts at 7,8, and $9 \mathrm{GHz}$, but not at any other harmonics. At the higher power levels, pronounced SW peaks are observed at all harmonics, although the signal at these three frequencies remains the most strongly affected.

A number of qualitatively different frequency regions can be observed based on the data taken at high laser power. At low frequency we find that the maximum BLS counts decrease exponentially with harmonic number 2-4. At intermediate harmonic numbers (5-8) the BLS counts increase rapidly and reach a strong maximum at a ferromagnetic resonance (FMR) frequency of about $8 \mathrm{GHz}$. For yet higher harmonics, the counts again fall, most precipitously between the $9^{\text {th }}$ and

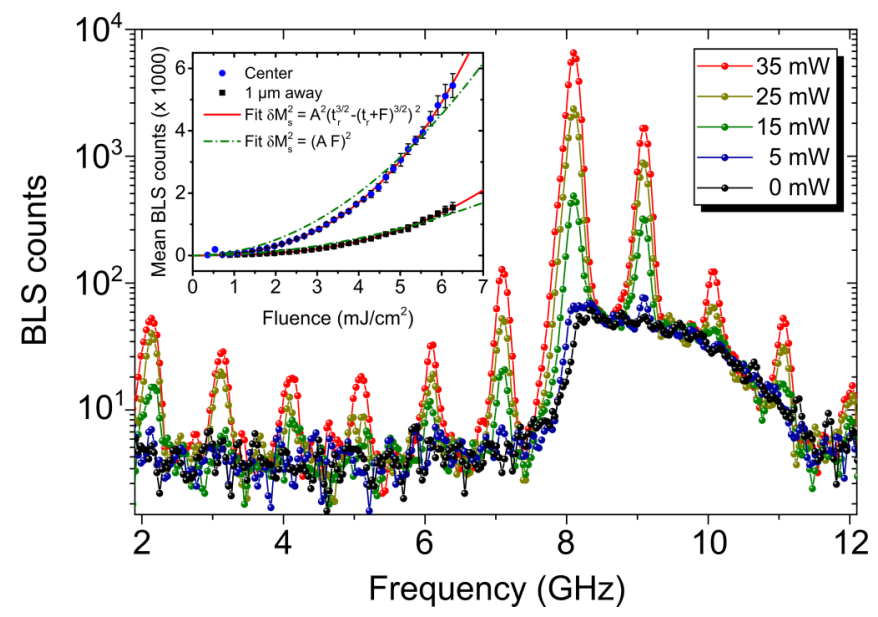

FIG. 3. Spin wave spectrum at different laser powers. BLS counts vs frequency in a field of $600 \mathrm{mT}$ for only thermal SWs (black) and at four different pump powers showing pronounced peaks at the harmonics of the $1 \mathrm{GHz}$ repetition rate. The inset shows the nonlinear increase of the BLS counts for the $8 \mathrm{GHz}$ mode with increasing pump fluence $(F)$ at the center of the pump spot and $1 \mu \mathrm{m}$ away together with two fits, one parabolic and the other based on Eq. (1). the $10^{\text {th }}$ harmonic, above which the impact of the fs laser remains limited compared to the thermal SWs. When the same data are taken with the location of the BLS probe separated $1 \mu \mathrm{m}$ away from the pump spot, the number of peaks is dramatically reduced with essentially only the 8 and $9 \mathrm{GHz}$ peaks surviving (not shown). We hence conclude that only at these two frequencies do the laser pulses generate propagating SWs of any magnitude; the magnetodynamics at all lower harmonics are consequently of a local or evanescent nature and the higher harmonics are more strongly damped out.

In the inset of Fig. 3 we plot the peak counts at $8 \mathrm{GHz}$ vs fs-laser fluence both at the pump spot and $1 \mu \mathrm{m}$ away. We have averaged the results from five different measurement spots in each case (both the pump and the probe lasers were moved to different locations). While the BLS counts clearly show a nonlinear, squarelike, dependence on fluence, the data are not well fitted by a parabola. Instead we develop a simple model where we incorporate a Bloch law dependence for how the magnitude of the demagnetization pulse depends on the effective magnon temperature.

We first assume that each pulse rapidly increases the temperature of the magnetic subsystem in linear proportion to the laser fluence, causing a rapid reduction of the magnetization $\delta M_{s}$ followed by a slower recovery. The temperature dependence of the magnetization should follow a Bloch $T^{3 / 2}$ law,

$$
\delta M_{s}=A\left[t_{r}^{3 / 2}-\left(t_{r}+F\right)^{3 / 2}\right],
$$

where $F$ is the laser fluence, and $A$ and $t_{r}$ are coefficients defined by the magnetization $M_{s}(T=0)$, the Curie and room temperatures, and the heating efficiency of the laser. As the SW amplitude should be linear in the demagnetization pulse $\left(\delta M_{s}\right)$ and the BLS signal should be proportional to the square of the SW amplitude, one can fit the BLS counts using the square of Eq. (1).

As seen in the inset of Fig. 3, this model is found to fit both curves exceptionally well, and much better than a simple parabola. We hence conclude that heating of the magnon population in direct proportion to the laser power describes the rapid demagnetization very well, and that BLS counts are a very sensitive probe to study the power dependence of rapid demagnetization.

\section{B. Spatial mapping of spin wave amplitude}

To study in detail the propagation characteristics of all excited SW modes, we measured the spatial extension of the spectra at a $600 \mathrm{mT}$ applied field by raster scanning over the film. Figures 4(a)-4(d) are the respective mode profiles of the $7,8,9$, and $10 \mathrm{GHz}$ peaks. The mode at $7 \mathrm{GHz}$ is clearly localized at the pump spot. At $8 \mathrm{GHz}$ the pump harmonic coincides approximately with the broadband ferromagnetic resonance peak and at $9 \mathrm{GHz}$ with higher wave vector SWs. High-amplitude SWs are then excited from the center and propagate in the direction perpendicular to the in-plane component of the applied field. At $10 \mathrm{GHz}$, we do not find any measurable evidence for SW propagation. As SW frequencies of $10 \mathrm{GHz}$ in a field of $0.6 \mathrm{~T}$ correspond to wave vectors just above the cutoff of the resolution of our BLS [see Fig. 2(a)] we cannot say for certain that no SW propagation takes place 

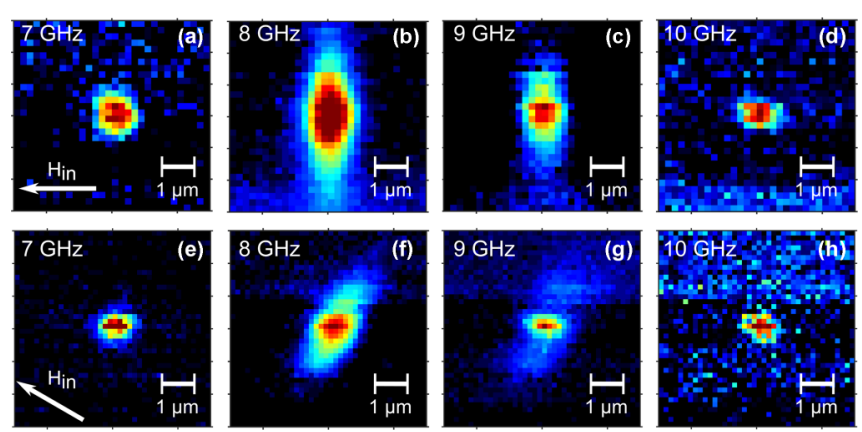

FIG. 4. 2D spatial profiles of the spin wave excitation. (a)-(d) Area maps of the spin wave intensities at 7, 8, 9, and $10 \mathrm{GHz}$. (e)-(h) show the same type of maps after the in-plane component of the applied field has been rotated 30 degrees clockwise.

at these high wave vectors. However, as the wave-vector cutoff for excitation and detection should depend similarly on spot size, and the spot size of the fs laser $(\sim 800 \mathrm{~nm})$ is larger than that of the BLS laser $(\sim 350 \mathrm{~nm})$, this is unlikely. Additionally, in Figs. 4(e)-4(h) we demonstrate that it is also possible to steer the propagation direction by rotating the in-plane component of the applied magnetic field.

\section{Micromagnetic simulations}

To gain further insight into the nature of the SW emission, we performed micromagnetic simulations at an applied external field value of $0.6 \mathrm{~T}$. The micromagnetic simulations were performed on a $5.12 \times 5.12 \mu \mathrm{m}^{2}$ permalloy film of $20 \mathrm{~nm}$ thickness using Mumax3 [41]. Periodic boundary conditions were used to suppress finite-size effects expected for this sample. A saturation magnetization $M_{s}$ of $781.75 \mathrm{kA} / \mathrm{m}$ extracted experimentally from thermally excited FMR, exchange stiffness $A$ of $11.3 \mathrm{pJ} / \mathrm{m}$, and damping of 0.01 are the parameters used in the simulations. As in the experiment, an external field was applied at an oblique angle of $82^{\circ}$.

To mimic the optical-pump effect spot used in the experiment, an instantaneous reduction of the saturation magnetization was followed by a slower recovery where the magnetization relaxes back to its original value, applied at a clock frequency of $1 \mathrm{GHz}$ in the form of subtracting or adding the demagnetization tensor corresponding to the magnetization state at both the demagnetized and the recovered states. The pump beam, as in the experiment, had a Gaussian profile with FWHM of $800 \mathrm{~nm}$.

The number of magnons per unit volume (density of a magnetic moment carried by spin waves), at a certain frequency $\omega$, was extracted from the results of simulations using
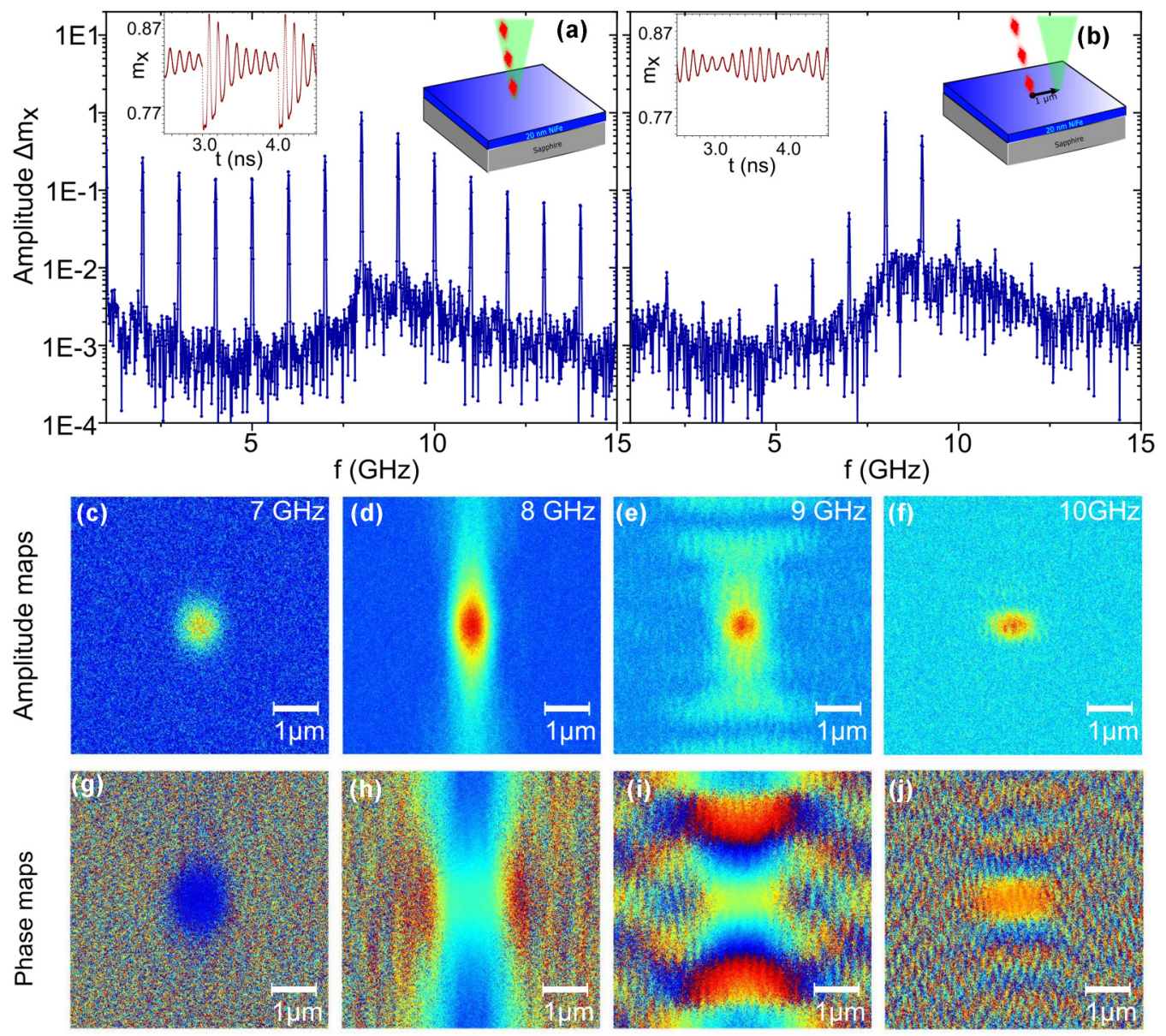

FIG. 5. Simulated spectral density of the magnetization dynamics at (a) the pump location, and (b) a distance $1 \mu \mathrm{m}$ from the pump location. Insets show the instantaneous temporal evolution of the magnetization. The simulated amplitude (c)-(f) and the phase profiles (g)-(j) at 7, 8, 9, and $10 \mathrm{GHz}$ are shown in the lower panels. 
Eq. (S19) in [42], $N(\omega)=\frac{M_{s}}{2 \mu_{B}} \mathbf{m}^{*}(\omega) \cdot\left[\mathbf{m}_{0} \times \mathbf{m}(\omega)\right]$, where $\mathbf{m}_{\mathbf{0}}$ is a unit vector, in the direction of the equilibrium magnetization, and $\mathbf{m}(\omega)$ the dynamic magnetization in Fourier space. Our micromagnetic simulations show almost perfect quadratic dependence of the magnon population at $8 \mathrm{GHz}$ on induced demagnetization $\delta M$.

The results were found to closely reproduce the overall behavior of the experimental spectra and the spatial profiles of the experimentally observed SW modes. Figure 5 shows the spectrum of the harmonics excited at the pump spot (a) and at a distance $1 \mu \mathrm{m}$ away (b). While the magnetization dynamics at the laser spot is dominated by the FMR response, all other harmonics of $1 \mathrm{GHz}$ are clearly present. At low frequencies, the amplitude decreases with harmonic number toward a shallow minimum at $n=4-5$ after which the amplitude again increases toward the FMR response; at frequencies above FMR the amplitude at the laser spot again decreases exponentially with $n$. As in the experiment, the spectral response is dramatically different $1 \mu \mathrm{m}$ away from the laser spot, where the FMR peak and the first propagating SW mode dominate entirely and all other peaks are strongly suppressed. The insets show time traces of the $x$ component of the magnetization at the two locations. While both time traces show a continuous SW intensity, the dynamics at the laser spot is more strongly affected by each pulse, whereas the response at $1 \mu \mathrm{m}$ away varies smoothly and exhibits beating from the sum of the two dominant spectral lines.

Figures 5(c)-5(j) show the spatial maps of the SW amplitude and phase at modes corresponding to 7, 8, 9, and $10 \mathrm{GHz}$. As observed in the experiment, the SWs at $7 \mathrm{GHz}$ correspond to a localized mode, as its frequency falls below the FMR. All higher frequencies correspond to propagating spin waves with wave vectors perpendicular to the in-plane component of the applied magnetic field.

\section{CONCLUSIONS AND OUTLOOK}

In conclusion, we have demonstrated, using a combined diffraction-limited BLS SW detection scheme with a diffraction-limited high repetition rate $(1 \mathrm{GHz})$ fs laser inducing rapid demagnetization, continuous coherent $\mathrm{SW}$ emission over a wide range of fields and frequencies. The highly efficient SW generation only occurs at higher harmonics of the $1 \mathrm{GHz}$ laser repetition rate, where the SWs that are in phase with the incoming laser pulses are coherently amplified, while all other SWs are left unaffected. The high sensitivity of our BLS microscope resolves spatial maps of both localized and propagating SWs. We have demonstrated that a specific SW wave vector can be coherently amplified, and its propagation steered in any direction by tuning the SW dispersion through the magnitude and direction of the applied magnetic field. In contrast to TR-MOKE measurements, where the signal increases linearly with laser power, we observe a nonlinear, stronger than square dependence of the BLS counts. The deviations from a square dependence can be fully accounted for by incorporating a Bloch law dependence of how the step in the demagnetization field relates to the instantaneous magnon temperature. Our results clearly demonstrate the versatility and benefits of using BLS microscopy for the study of rapid demagnetization and will enable a wide range of additional photomagnonic studies of magnetic thin films and magnonic and spintronic devices. While our study used a single excitation spot, it will be straightforward to extend to multiple spots and/or shape the SW excitation region using optical techniques such as spatial light modulation. The possibility of adding sustained SW excitation to the studies of spin transfer torque and spin Hall effect driven nanoscale devices is particularly intriguing as SWs play a key role in their operation.

\section{ACKNOWLEDGMENTS}

This work was supported by the Knut and Alice Wallenberg Foundation, the Swedish Research Council, and the Horizon 2020 research and innovation program (ERC Advanced Grant No. 835068 “TOPSPIN").

S.M., A.A.A., and A.A. contributed equally to this work.
[1] V. V. Kruglyak, S. O. Demokritov, and D. Grundler, J. Phys. D 43, 264001 (2010).

[2] Magnonics: From Fundamentals to Applications, edited by S. O. Demokritov and A. N. Slavin, Topics in Applied Physics (Springer Nature, Switzerland AG, 2013).

[3] S. Neusser and D. Grundler, Adv. Mater. 21, 2927 (2009).

[4] A. V. Chumak, V. Vasyuchka, A. Serga, and B. Hillebrands, Nat. Phys. 11, 453 (2015).

[5] H. Yu, O. d'Allivy Kelly, V. Cros, R. Bernard, P. Bortolotti, A. Anane, F. Brandl, F. Heimbach, and D. Grundler, Nat. Commun. 7, 11255 (2016).

[6] P. Wolf, J. Appl. Phys. 32, S95 (1961).

[7] A. K. Ganguly and D. C. Webb, IEEE Trans. Microwave Theory Tech. 23, 998 (1975).

[8] V. E. Demidov, S. O. Demokritov, K. Rott, P. Krzysteczko, and G. Reiss, Appl. Phys. Lett. 92, 232503 (2008).

[9] V. E. Demidov, S. Urazhdin, and S. O. Demokritov, Nat. Mater. 9, 984 (2010).
[10] S. Bonetti, V. Tiberkevich, G. Consolo, G. Finocchio, P. Muduli, F. Mancoff, A. Slavin, and J. Åkerman, Phys. Rev. Lett. 105, 217204 (2010).

[11] M. Madami, S. Bonetti, G. Consolo, S. Tacchi, G. Carlotti, G. Gubbiotti, F. B. Mancoff, M. A. Yar, and J. Åkerman, Nat. Nanotechnol. 6, 635 (2011).

[12] T. Chen, R. K. Dumas, A. Eklund, P. K. Muduli, A. Houshang, A. A. Awad, P. Dürrenfeld, B. G. Malm, A. Rusu, and J. Åkerman, Proc. IEEE 104, 1919 (2016).

[13] A. Houshang, E. Iacocca, P. Dürrenfeld, S. R. Sani, J. Åkerman, and R. K. Dumas, Nat. Nanotechnol. 11, 280 (2016).

[14] P. Dürrenfeld, A. A. Awad, A. Houshang, R. K. Dumas, and J. Åkerman, Nanoscale 9, 1285 (2017).

[15] A. Houshang, R. Khymyn, H. Fulara, A. Gangwar, M. Haidar, S. R. Etesami, R. Ferreira, P. P. Freitas, M. Dvornik, R. K. Dumas, and J. Åkerman, Nat. Commun. 9, 4374 (2018). 
[16] B. Divinskiy, V. E. Demidov, S. Urazhdin, R. Freeman, A. B. Rinkevich, and S. O. Demokritov, Adv. Mater. 30, 1802837 (2018).

[17] H. Fulara, M. Zahedinejad, R. Khymyn, A. A. Awad, S. Muralidhar, M. Dvornik, and J. Åkerman, Sci. Adv. 5, eaax8467 (2019).

[18] E. Beaurepaire, J.-C. Merle, A. Daunois, and J.-Y. Bigot, Phys. Rev. Lett. 76, 4250 (1996).

[19] B. Koopmans, G. Malinowski, F. Dalla Longa, D. Steiauf, M. Fähnle, T. Roth, M. Cinchetti, and M. Aeschlimann, Nat. Mater. 9, 259 (2010).

[20] D. Rudolf, C. La-O-Vorakiat, M. Battiato, R. Adam, J. M. Shaw, E. Turgut, P. Maldonado, S. Mathias, P. Grychtol, H. T. Nembach, T. J. Silva, M. Aeschlimann, H. C. Kapteyn, M. M. Murnane, C. M. Schneider, and P. M. Oppeneer, Nat. Commun. 3, 1037 (2012).

[21] C.-H. Lambert, S. Mangin, B. S. D. C. S. Varaprasad, Y. K. Takahashi, M. Hehn, M. Cinchetti, G. Malinowski, K. Hono, Y. Fainman, M. Aeschlimann, and E. E. Fullerton, Science 345, 1337 (2014).

[22] M. van Kampen, C. Jozsa, J. T. Kohlhepp, P. LeClair, L. Lagae, W. J. M. de Jonge, and B. Koopmans, Phys. Rev. Lett. 88, 227201 (2002).

[23] M. Vomir, L. H. F. Andrade, L. Guidoni, E. Beaurepaire, and J.-Y. Bigot, Phys. Rev. Lett. 94, 237601 (2005).

[24] A. V. Kimel, A. Kirilyuk, A. Tsvetkov, R. V. Pisarev, and T. Rasing, Nature (London) 429, 850 (2004).

[25] F. Hansteen, A. Kimel, A. Kirilyuk, and T. Rasing, Phys. Rev. Lett. 95, 047402 (2005).

[26] F. Hansteen, A. Kimel, A. Kirilyuk, and T. Rasing, Phys. Rev. B 73, 014421 (2006).

[27] A. V. Kimel, A. Kirilyuk, P. A. Usachev, R. V. Pisarev, A. M. Balbashov, and T. Rasing, Nature (London) 435, 655 (2005).

[28] Y. Au, M. Dvornik, T. Davison, E. Ahmad, P. S. Keatley, A. Vansteenkiste, B. Van Waeyenberge, and V. V. Kruglyak, Phys. Rev. Lett. 110, 097201 (2013).
[29] S.-J. Yun, C.-G. Cho, and S.-B. Choe, Appl. Phys. Express 8, 063009 (2015).

[30] S. Iihama, Y. Sasaki, A. Sugihara, A. Kamimaki, Y. Ando, and S. Mizukami, Phys. Rev. B 94, 020401(R) (2016).

[31] A. Kamimaki, S. Iihama, Y. Sasaki, Y. Ando, and S. Mizukami, Phys. Rev. B 96, 014438 (2017).

[32] T. Satoh, Y. Terui, R. Moriya, B. A. Ivanov, K. Ando, E. Saitoh, T. Shimura, and K. Kuroda, Nat. Photon. 6, 662 (2012).

[33] M. Deb, M. Vomir, J. L. Rehspringer, and J. Y. Bigot, Appl. Phys. Lett. 107, 252404 (2015).

[34] D. Bossini, S. Dal Conte, Y. Hashimoto, A. Secchi, R. V. Pisarev, T. Rasing, G. Cerullo, and A. V. Kimel, Nat. Commun. 7, 10645 (2016).

[35] C. Berk, F. Ganss, M. Jaris, M. Albrecht, and H. Schmidt, IEEE J. Sel. Top. Quantum Electron. 25, 1 (2019).

[36] M. Jäckl, V. I. Belotelov, I. A. Akimov, I. V. Savochkin, D. R. Yakovlev, A. K. Zvezdin, and M. Bayer, Phys. Rev. X 7, 021009 (2017).

[37] I. V. Savochkin, M. Jäckl, V. I. Belotelov, I. A. Akimov, M. A. Kozhaev, D. A. Sylgacheva, A. I. Chernov, A. N. Shaposhnikov, A. R. Prokopov, V. N. Berzhansky, D. R. Yakovlev, A. K. Zvezdin, and M. Bayer, Sci. Rep. 7, 5668 (2017).

[38] U. Atxitia, O. Chubykalo-Fesenko, J. Walowski, A. Mann, and M. Münzenberg, Phys. Rev. B 81, 174401 (2010).

[39] J. Mendil, P. Nieves, O. Chubykalo-Fesenko, J. Walowski, T. Santos, S. Pisana, and M. Münzenberg, Sci. Rep. 4, 3980 (2014).

[40] T. Sebastian, K. Schultheiss, B. Obry, B. Hillebrands, and H. Schultheiss, Front. Phys. 3, 1 (2015).

[41] A. Vansteenkiste, J. Leliaert, M. Dvornik, M. Helsen, F. Garcia Sanchez, and B. Van Waeyenberge, AIP Adv. 4, 107133 (2014).

[42] O. Dzyapko, I. Lisenkov, P. Nowik-Boltyk, V. E. Demidov, S. O. Demokritov, B. Koene, A. Kirilyuk, T. Rasing, V. Tiberkevich, and A. Slavin, Phys. Rev. B 96, 064438 (2017). 